\title{
(13-polyunsaturated fatty acids induce cell death through apoptosis and autophagy in glioblastoma cells: In vitro and in vivo
}

\author{
SOYEON KIM ${ }^{1}$, KAIPENG JING ${ }^{4}$, SOYEON SHIN ${ }^{1}$, SOYEON JEONG ${ }^{1}$, SEUNG-HYEON HAN ${ }^{1,2}$, \\ HEYRIM OH $^{1}$, YOON-SEON YOO ${ }^{1,2}$, JEONGSU HAN ${ }^{1}$, YOUNG-JOO JEON ${ }^{1,2}$, JUN-YOUNG HEO ${ }^{1,2}$, \\ GI-RYANG KWEON ${ }^{1,2}$, SEUNG-KIEL PARK ${ }^{1}$, JONG-IL PARK ${ }^{1}$, TONG WU ${ }^{5}$ and KYU LIM ${ }^{1-3}$ \\ Departments of ${ }^{1}$ Biochemistry, ${ }^{2}$ Medical Science, School of Medicine, and ${ }^{3}$ Cancer Research Institute, \\ Chungnam National University, Daejeon, Republic of Korea; ${ }^{4}$ Stem Cell Research and Cellular Therapy Center, \\ Affiliated Hospital of Guangdong Medical College, Zhanjiang, Guangdong, P.R. China; ${ }^{5}$ Department of \\ Pathology and Laboratory Medicine, Tulane University School of Medicine, New Orleans, LA, USA
}

Received June 27, 2017; Accepted October 30, 2017

DOI: 10.3892/or.2017.6101

\begin{abstract}
Among brain tumors, glioblastoma (GBM) is the most aggressive type and is associated with the lowest patient survival rate. Numerous lines of evidence have established that omega-3-polyunsaturated fatty acids ( $\omega 3$-PUFAs) have potential for the prevention and therapy of several types of cancers. Docosahexaenoic acid (DHA), an $\omega 3$-PUFA, was reported to inhibit growth and induce apoptotic and autophagic cell death in several cancer cell lines; however, its effects on GBM cells are still unknown. In the present study, we examined the cytotoxic effect of DHA on the GBM cell lines, D54MG, U87MG, U251MG and GL261. Treatment of GBM cells with DHA induced PARP cleavage, increased the population of sub-G1 cells, and increased the number of TUNEL-positive cells, which are all indicative of apoptosis. Furthermore, treatment of GBM cells with DHA resulted in a significant increase in autophagic activity, as revealed by increased LC3-II levels, GFP-LC3 puncta, and autophagic flux activation, accompanied by activation of 5'-AMP-activated protein kinase (AMPK) and decreases in phosphorylated Akt (p-AktSer ${ }^{473}$ ) levels and mTOR activity. In vivo, endogenous expression of Caenorhabditis elegans $\omega 3$-desaturase, which converts $\omega 6$-PUFAs to $\omega 3$-PUFAs, in fat-1 transgenic mice yielded a significant decrease in tumor volume following subcutaneous injection of mouse glioma cells (GL261), when compared with wild-type mice. TUNEL-positive cell numbers and LC3-II levels were elevated in tumor tissue from the fat- 1 transgenic mice compared with tumor tissue from the wild-type mice. In addition, p-Akt levels were decreased and p-AMPK levels
\end{abstract}

Correspondence to: Professor Kyu Lim, Department of Biochemistry, School of Medicine, Chungnam National University, Daejeon 301-747, Republic of Korea

E-mail: kyulim@cnu.ac.kr

Key words: autophagy, DHA, glioblastoma, omega-3 fatty acid, mTOR were increased in tumor tissue from the fat-1 transgenic mice. These results indicate that $\omega 3$-PUFAs induce cell death through apoptosis and autophagy in GBM cells; thus, it may be possible to use $\omega 3$-PUFAs as chemopreventive and therapeutic agents for GBM.

\section{Introduction}

Glioblastoma (GBM) is the most aggressive and deadliest brain malignancy in adults. According to the latest statistics from the United States National Cancer Institute, it is estimated that 23,000 Americans will be diagnosed with brain and other nervous system cancers in 2017 and 16,700 will die from these cancers (1). Gliomas are divided into four grades, of which grade four or GBM is the most aggressive and has the highest incidence (2). GBM progression cannot be controlled by surgical resection, radiotherapy, or chemotherapy, which results in a median survival time of less than 15 months (3). Thus, new therapeutic approaches are needed.

Omega3-polyunsaturated fatty acids ( $\omega 3$-PUFAs) and $\omega 6$-polyunsaturated fatty acids ( $\omega 6$-PUFAs) are essential nutrients for good health. $\omega 3$-PUFAs are long-chain fatty acids that have a double bond between the third and fourth carbon atoms from the methyl end of the carbon chain. The Western diet contains a high amount of $\omega 6$-PUFAs, with an $\omega 6$-PUFA to $\omega 3$-PUFA ratio of approximately 50:1 (4). A high $\omega 6$-PUFA to $\omega 3$-PUFAs ratio contributes to the pathogenesis of many diseases, including cancer and inflammatory diseases, whereas increased levels of $\omega 3$-PUFAs (a low $\omega 6$-PUFA to $\omega 3$-PUFA ratio) have suppressive effects on these diseases (5). For this reason, nutritionists recommend a diet with an optimal $\omega 6$-PUFA to $\omega 3$-PUFA ratio of 1:1 (6). We previously demonstrated in numerous studies that $\omega 3$-PUFAs, docosahexaenoic acid (DHA) in particular, induce cell death in cancer cells through several mechanisms, including inhibition of Wnt/ $\beta$-catenin signaling (7-9), activation of mitogen-activated protein kinases (10), inhibition of Cox-2/PGE2 signaling $(7,8,11)$ and inhibition of NF- $\kappa \mathrm{B}$ signaling (11). Recently, we reported that DHA induces autophagy through p53/AMPK/mTOR signaling (12) and that 
autophagy induction enhances apoptosis in human cancer cells (12-14). More recently, DHA was found to induce degradation of HPV E6/E7 oncoproteins by activating the ubiquitin-proteasome system (15). However, the anticancer activity of DHA in GBM cells has not been studied intensively. In addition, the molecular mechanisms of the anticancer activity of DHA are not fully understood. Therefore, in the present study, we investigated the mechanisms underlying the anticancer activity of DHA on GBM in vitro and in vivo.

\section{Materials and methods}

Glioma cell lines and reagent treatment. Three human (D54MG, U87MG and U251MG) and mouse (GL261) glioma cell lines were used in the present study. D54MG and GL261 cell lines were obtained from Dr Yancie Gillespie (University of AlabamaBirmingham, Birmingham, AL). To note, the D54MG cell line is misidentified according to the following database: http://iclac. org/wp-content/uploads/Cross-Contaminations-v8_0.pdf. This cell line is contaminated with another human glioblastoma cell line (A-172). U87MG, U251MG and GL261 cells were maintained in Dulbecco's modified Eagle's medium (DMEM; Gibco, Grand Island, NY, USA), and D54MG was grown in RPMI1640 medium (Gibco) supplemented with $10 \%$ fetal bovine serum (FBS; Gibco) and antibiotics (penicillin 10,000 U/ml and streptomycin $10,000 \mu \mathrm{g} / \mathrm{ml} ;$ Gibco) in $5 \% \mathrm{CO}_{2}$ at $37^{\circ} \mathrm{C}$. Absolute ethanol was used to dissolve DHA (Cayman Chemical, Ann Arbor, MI, USA). The following antibodies were used in the present study: poly(ADP-ribose) polymerase (PARP) (\#9542, Rabbit, Polyclonal, 1:2,000), LC3B (\#3868, Rabbit IgG, Monoclonal, 1:5,000), Akt (\#9272, Rabbit, Polyclonal, 1:2,000), p-Akt(Ser473) (\#4060, Rabbit IgG, Monoclonal, 1:2,000), mTOR (\#2972, Rabbit, Polyclonal, 1:1,000), p-mTOR(Ser2448) (\#2971, Rabbit, Polyclonal, 1:1,000), AMPK (\#2532, Rabbit, Polyclonal, 1:2,000) and p-AMPK(Thr172) (\#2535, Rabbit IgG, Monoclonal, 1:2,000). All antibodies were purchased from Cell Signaling Technology (Beverly, MA, USA). Goat anti-rabbit and goat anti-mouse secondary antibodies were purchased from Calbiochem (Billerica, MA, USA).

MTT assay. Cell viability was determined using thiazolyl blue tetrazolium bromide (MTT; Sigma-Aldrich, St. Louis, MO, USA). D54MG, U87MG, U251MG and GL261 cells were seeded onto 96-well tissue culture plates and incubated at $37^{\circ} \mathrm{C}$ for $18 \mathrm{~h}$. The cells were incubated with serum-free media for $24 \mathrm{~h}$ at $37^{\circ} \mathrm{C}$, after which the cells were treated with the indicated concentrations of DHA for another $24 \mathrm{~h}$ whereas the D54MG cells were incubated for $6 \mathrm{~h}$. The cells were then incubated with $0.005 \mathrm{mg} / \mathrm{ml}$ of MTT dissolved in serum-free medium for $1 \mathrm{~h}$ at $37^{\circ} \mathrm{C}$. After a 1-h incubation, the formazan product, which is indicated by dark blue water-insoluble crystals, was formed in the event the cells were alive. These crystals were dissolved in $100 \mu \mathrm{l}$ dimethyl sulfoxide (DMSO), the absorbance was then measured at $570 \mathrm{~nm}$, and cell viability was expressed as a ratio vs. the untreated control cells. Each experiment was performed in triplicate.

Western blot analysis. Cells extracts were prepared from $100-\mathrm{mm}$ tissue culture plates. Cell pellets were lysed in cell lysis buffer and then sonicated for $20 \mathrm{sec}$. The supernatants were prepared by centrifugation at $17,000 \mathrm{rpm}$ at $4^{\circ} \mathrm{C}$ for 20 min. Cell lysates $(30 \mu \mathrm{g})$ were separated using 6-15\% sodium dodecyl sulfate-polyacrylamide gel electrophoresis (SDS-PAGE). The separated proteins were then transferred to a PVDF membrane (Millipore, Billerica, MA, USA), and the membranes were blocked with 5\% skim milk in TBS/T for $1 \mathrm{~h}$ at room temperature. The blots were incubated with the diluted primary antibodies overnight at $4^{\circ} \mathrm{C}$, followed by incubation with goat anti-rabbit peroxidase-conjugated secondary antibody. The bands were then visualized with the ECL western blotting detection system (WBKLS0500, Millipore) according to the manufacturer's instructions.

Flow cytometry assay. For the flow cytometry cell sorting (FACS) assay, both floating and attached cells were collected after the drug treatment, washed in phosphate-buffered saline (PBS), fixed with $70 \%$ ethanol for $24 \mathrm{~h}$, treated with $500 \mu \mathrm{g} / \mathrm{ml}$ of RNase A (Sigma-Aldrich), and then stained with $50 \mu \mathrm{g} / \mathrm{ml}$ propidium iodide (PI; Sigma-Aldrich) for $10 \mathrm{~min}$ at $37^{\circ} \mathrm{C}$. DNA staining with PI was analyzed with a FACSCalibur flow cytometer (BD Biosciences, San Diego, CA, USA).

TUNEL assay. For the TUNEL assay in vitro, D54MG cells were seeded onto glass coverslips in 24-well plates and then grown for $24 \mathrm{~h}$. The cells were then incubated with serum-free media for $24 \mathrm{~h}$ at $37^{\circ} \mathrm{C}$, and then the cells were treated with $30 \mu \mathrm{M}$ DHA for another $2 \mathrm{~h}$. Cells were washed with 1X PBS and processed with TUNEL using the DeadEnd Fluorometric TUNEL system (Promega, Fitchburg, WI, USA). Cells were mounted with mounting solution (Dako A/S, Copenhagen, Denmark) with DAPI. The number of TUNEL-positive (green) and DAPIpositive cells (blue nuclear stain) was visually counted. All samples were analyzed with at least two biological replicates, and three images from each replicate were captured using a 10X objective for counting the TUNEL- and DAPI-positive cells. The percentage of TUNEL-positive cells was calculated as (number of TUNEL-positive cells/total number of cells) x 100.

Transfection. Cells grown to $80 \%$ confluency were switched to and incubated with serum-free media for $24 \mathrm{~h}$. Transient transfection was then performed with the GFP-LC3 expression vector (a kind gift from Dr Tamotsu Yoshimori, National Institute of Genetics, Mishima, Japan) as recommended by the vendor. After $24 \mathrm{~h}$ of transfection, the cells were exposed to the indicated concentrations of DHA for $1 \mathrm{~h}$. The transfected cells were then observed under a fluorescence microscope, and fluorescence imaging was performed to detect the punctate pattern of GFP-LC3B.

Tumorigenicity experiments. Transgenic (tg) mice were kindly provided by Dr J.X. Kang (Harvard University, Cambridge, MA, USA). Control C57BL/6 mice were purchased from Central Lab Animal Inc., Seoul, Korea. The control and fat-1 tg mice were kept under specific pathogen-free conditions and received care according to the guidelines of the Institutional Animal Care and Use Committee of Chungnam National University which approved the protocol of the animal research. Both control and fat- 1 tg mice were 6-week-old male mice. Each mouse was subcutaneously injected with $2 \times 10^{6}$ cells (mouse glioma GL261 cells) in a total volume of $100 \mu 1$. The 

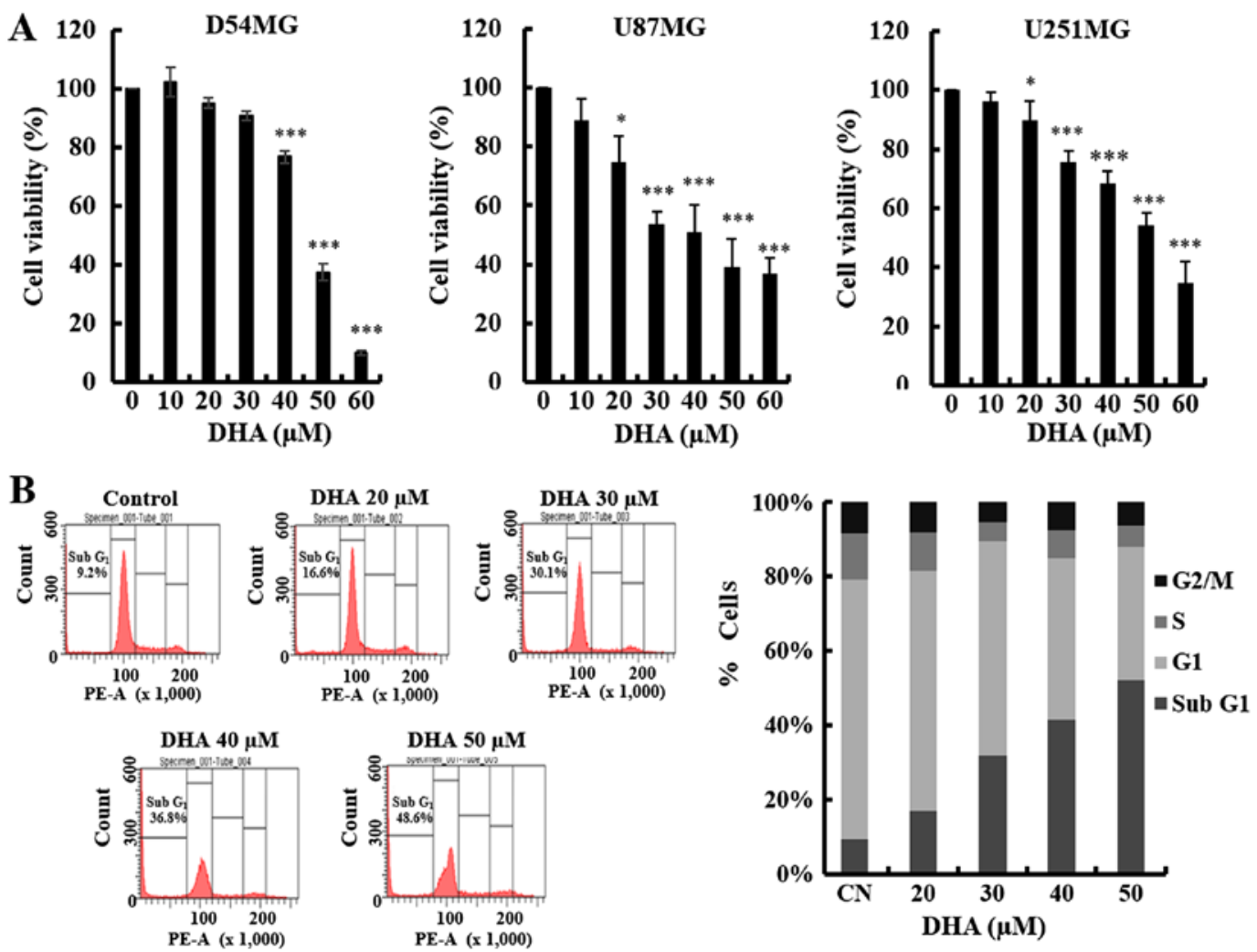

Figure 1. DHA inhibits cell viability and induces apoptosis in human cancer cells. (A) DHA reduced the viability of D54MG, U87MG and U251MG cells in a dose-dependent manner. Cells were exposed to the indicated doses of DHA for $24 \mathrm{~h}$ and the cell viability was measured using an MTT assay. Each bar represents the mean of three determinations. Each experiment was repeated three times. ${ }^{*} \mathrm{P}<0.05,{ }^{* * *} \mathrm{P}<0.001$. (B) DHA increases the number of D54MG cells in the sub-G1 phase. D54MG cells were seeded and treated with the indicated doses of DHA for $24 \mathrm{~h}$. The cell cycle distribution of DHA-treated cells was analyzed by flow cytometry as described in Materials and methods. Data were analyzed using FlowJo software. DHA, docosahexaenoic acid.

day of implantation was designated as day 0 . The tumor size was measured with a caliper every other day. The tumor size was calculated as length $\mathrm{x}$ wide, and the tumor volume was calculated as $0.5 \mathrm{x}$ length $\mathrm{x}$ (width) ${ }^{2}$.

Immunohistochemistry. After deparaffinization and antigen retrieval, the tissues were blocked with Dako Protein Block, stained with TUNEL using the DeadEnd Fluorometric TUNEL System (Promega), LC3B (1:3,000; Cell Signaling, Technology), p-Akt ${ }^{\mathrm{S} 473}$ (1:50; Cell Signaling, Technology), and p-AMPK ${ }^{\mathrm{T} 172}$ (1:100; Cell Signaling, Technology) primary antibodies, visualized using an anti-rabbit IgG conjugated secondary antibody with Texas Red and counterstained with DAPI. These fluorescently stained tissues were observed under a fluorescence microscope as previously described using the DP Controller software (Olympus) for image acquisition. Final images were a non-contrast-adjusted merge of the two channels.

Statistical analysis. Statistical analyses were performed as recommended by independent analysis. These included the unpaired Student's t-test. All values are expressed as mean \pm SD and statistical significance was indicated by $\mathrm{P}<0.05$ $\left({ }^{*} \mathrm{P}<0.05,{ }^{* *} \mathrm{P}<0.01\right.$ and ${ }^{* * *} \mathrm{P}<0.001$, respectively).

\section{Results}

DHA induces a cytotoxic effect on GBM cells. To investigate the effects of DHA on the growth of GBM cell lines, D54MG,
U87MG, U251MG and GL261, the cells were incubated with various concentrations of DHA for $24 \mathrm{~h}$, or $6 \mathrm{~h}$ in the case of D54MG cells. DHA decreased the viability of each GBM cell line in a dose-dependent manner, as determined by the MTT assay (Fig. 1A). A decrease in cell viability was observed at DHA concentrations of $20 \mu \mathrm{M}$ or greater. These findings support the hypothesis that DHA exerts a significant influence on GBM cell proliferation.

DHA induces apoptosis and autophagy in GBM cells. According to recent reports, DHA induces apoptosis in various types of cancer cells by activating both intrinsic and extrinsic pathways $(16,17)$. To assess whether DHA also induces apoptosis in GBM cells, we first analyzed the sub-G1 population of DHA-treated D54MG cells by flow cytometry. As shown in Fig. 1B, DHA induced an increase in the proportion of cells in the sub-G1 phase. To determine whether the observed reduction in cell viability was caused by apoptosis, we assessed the ability of DHA to induce apoptosis in human GBM cells. DHA led to proteolytic cleavage of poly(ADPribose) polymerase (PARP), which is widely used as an apoptosis marker (Fig. 2A). Furthermore, TUNEL assay was performed to detect apoptotic nuclear DNA breaks in D54MG cells; after DHA treatment, the number of TUNEL-positive cells increased significantly compared with the untreated control, in which no apoptotic activity was observed (Fig. 2B). These results demonstrated that DHA induced apoptosis in GBM cells. 


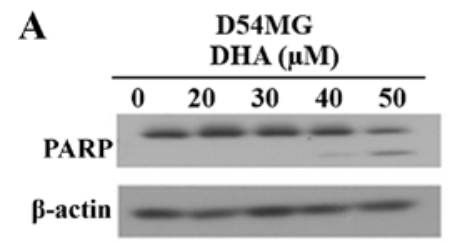

B

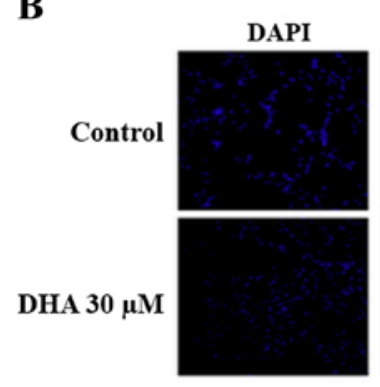

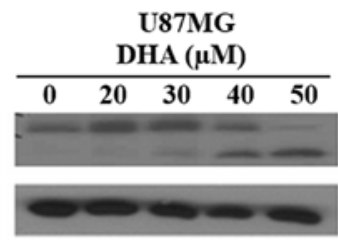

TUNEL

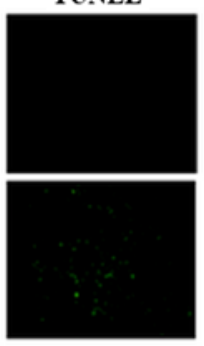

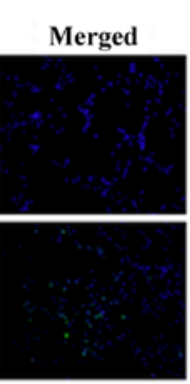
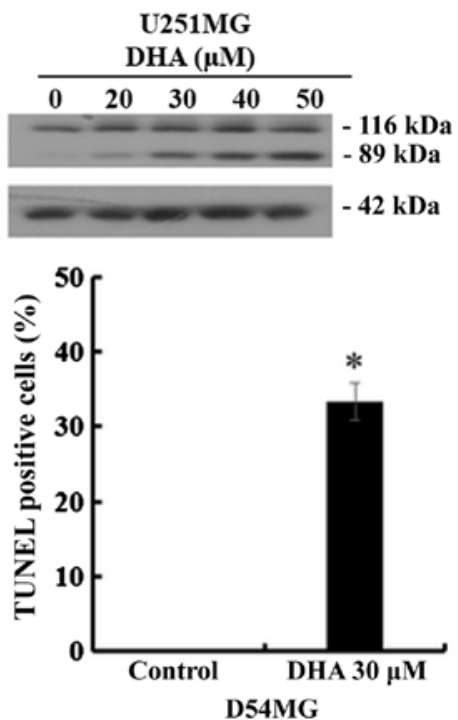

Figure 2. DHA induces apoptosis. (A) D54MG, U87MG and U251MG cells were incubated with the indicated doses of DHA for $24 \mathrm{~h}$. The cells were then harvested, and western blot analysis was performed with anti-PARP and anti-actin antibodies. (B) DHA increased the number of TUNEL-positive cells. D54MG cells were plated in a 12-well plate containing glass coverslips and then treated with $30 \mu \mathrm{M}$ DHA for $6 \mathrm{~h}$. Following treatment, apoptosis was detected using the DeadEnd Fluorometric TUNEL System. Left panel, representative fluorescence microscopy images (scale bar, $200 \mu \mathrm{m}$ ). Right panel, the percentage of TUNEL-positive cells in the presence or absence of DHA was expressed relative to the total number of DAPI-stained nuclei. TUNEL-positive cells were counted in three different fields and the numbers averaged; ${ }^{*} \mathrm{P}<0.05$. DHA, docosahexaenoic acid.

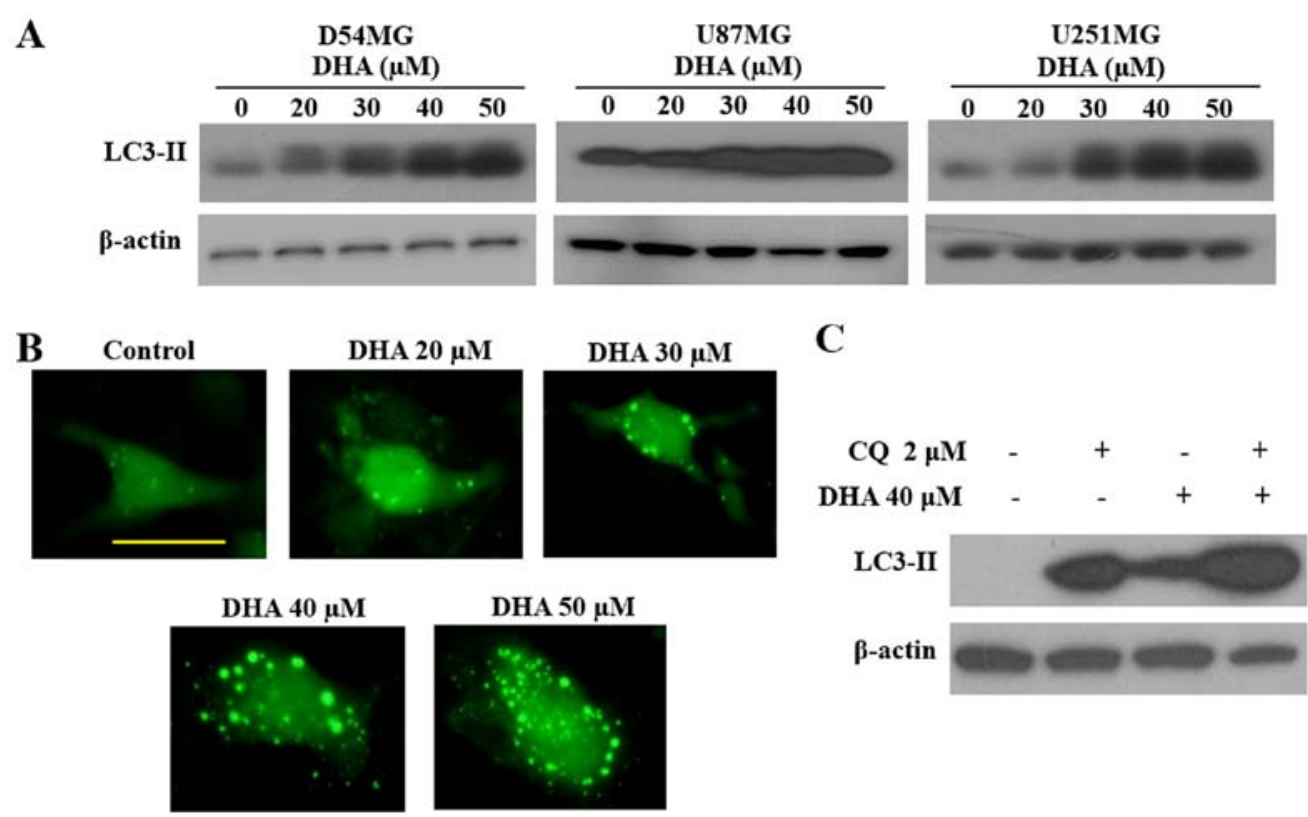

Figure 3. DHA induces autophagy. (A) DHA increased the expression of LC3-II in a dose-dependent manner. D54MG, U87MG and U251MG cells were exposed to the indicated doses of DHA for $24 \mathrm{~h}$. Protein lysates were then prepared, separated on SDS polyacrylamide gels and immunoblotted with antibodies against LC3-II and $\beta$-actin. (B) Formation of GFP-LC3 puncta in DHA-treated D54MG cells. The cells were transfected with a GFP-LC3 plasmid and then exposed to the indicated doses of DHA for another $24 \mathrm{~h}$ before counterstaining with DAPI. Representative fluorescence microscopy images are shown (scale bar, $20 \mu \mathrm{m}$ ). (C) DHA-induced autophagy increased D54MG cell death. D54MG cells were incubated for $1 \mathrm{~h}$ in the presence ( $2 \mu \mathrm{M})$ or absence of CQ before incubation with $40 \mu \mathrm{M}$ DHA for $24 \mathrm{~h}$. Cell lysates were prepared and examined by western blotting. CQ, chloroquine; DHA, docosahexaenoic acid.

To evaluate autophagy as another possible mechanism for induction of GBM cell death by DHA, we next performed autophagic cell death experiments. DHA treatment increased the level of LC3B-II, an autophagy marker, in a dose-dependent manner (Fig. 3A). This observation was confirmed in DHA-treated D54MG cells transfected with GFP-LC3B, which showed a greater number of GFP-LC3B puncta compared with untreated cells (Fig. 3B). Moreover, autophagic flux assays in cells pretreated with chloroquine, an inhibitor of lysosomal acidification, provided further confirmation that DHA treatment increased LC3-II expression (Fig. 3C). Altogether, these observations indicate that DHA induces autophagy in GBM cells.

DHA inhibits mTOR signaling through inhibition of Akt and activation of AMPK in GBM cells. The mTOR pathway is one of the most important pathways that regulate both apoptosis 


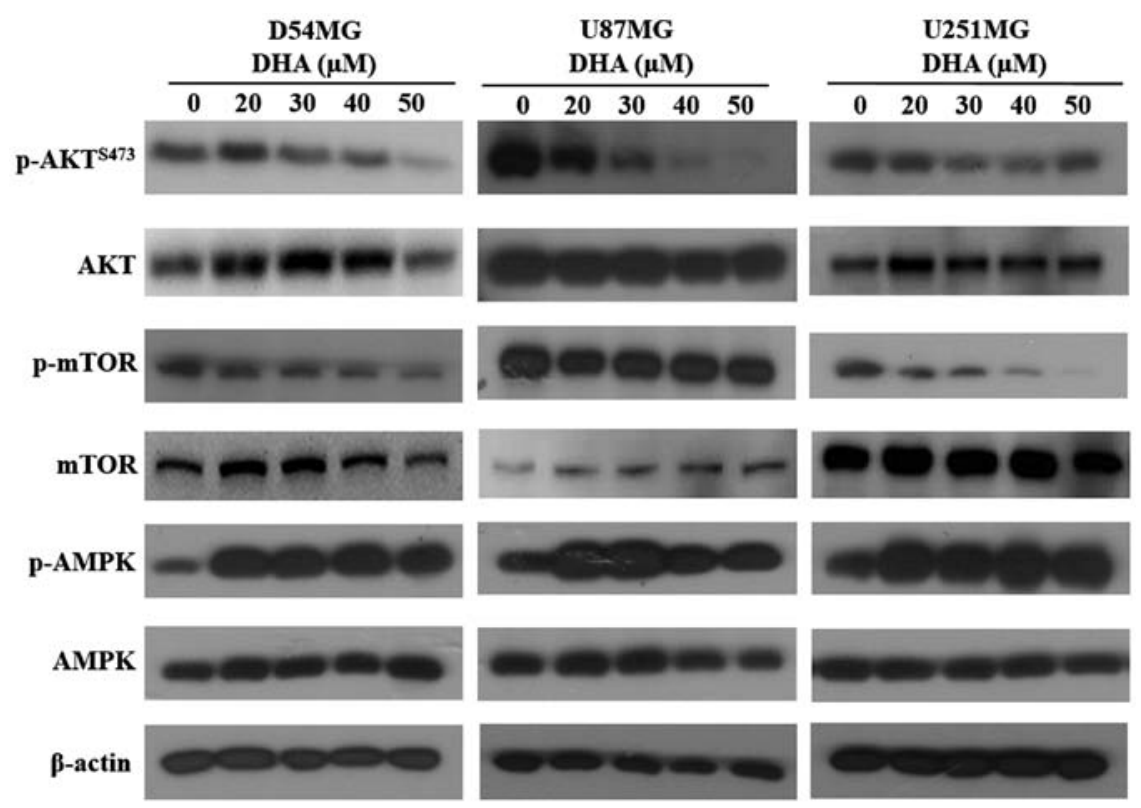

Figure 4. DHA-induced autophagy is related to the downregulation of mTOR signaling in GBM cells. DHA downregulated mTOR signaling in a dosedependent manner. D54MG, U87MG and U251MG cells were incubated with the indicated doses of DHA for $24 \mathrm{~h}$ and then subjected to western blot analysis with antibodies against phospho-mTOR, phospho-Akt, Akt, phospho-AMPK, AMPK and $\beta$-actin. DHA, docosahexaenoic acid.

$\mathbf{A}$

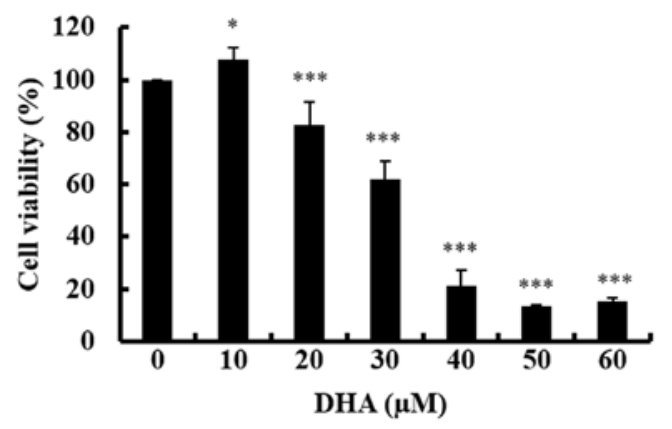

B
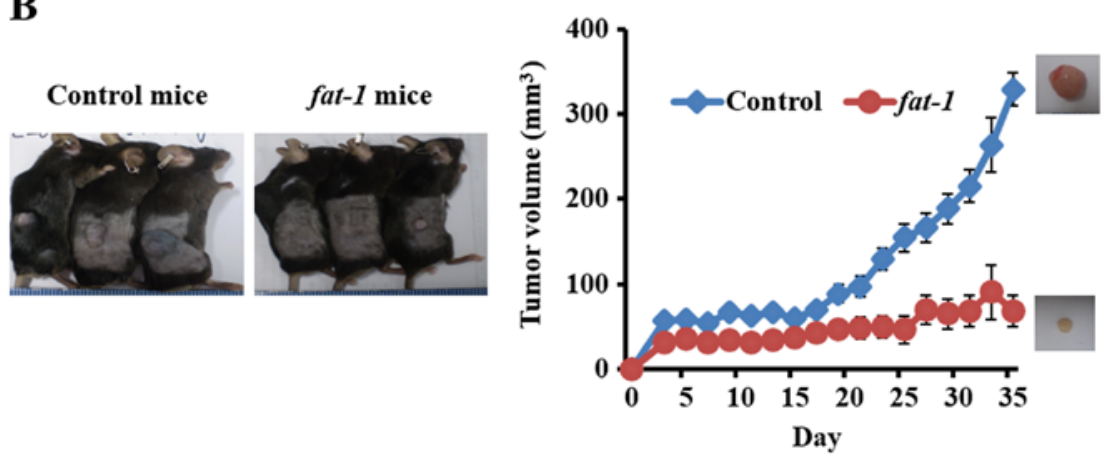

Figure 5. $\omega 3$-PUFA-rich environment suppresses tumor growth in vivo. (A) DHA reduces the viability of GL261 cells in a dose-dependent manner. Cells were exposed to the indicated doses of DHA for $24 \mathrm{~h}$, and the cell viability was measured using an MTT assay. Each bar represents the mean of three determinations. Each experiment was repeated three times; ${ }^{*} \mathrm{P}<0.05,{ }^{* * *} \mathrm{P}<0.001$. (B) Effect of $\omega 3$-PUFA on tumorigenicity. GL261 cells $\left(2 \times 10^{6}\right.$ cells) were injected subcutaneously into the flanks of wild-type and fat- 1 tg mice. Tumor volume was monitored every other day for 33 days. Tumor volume was calculated as described in Materials and methods. DHA, docosahexaenoic acid; $\omega 3$-PUFA, omega-3-polyunsaturated fatty acid.

and autophagy. This pathway is also directly linked to the Akt survival pathway, leading us to question whether the simultaneous induction of apoptosis and autophagy by DHA in GBM cells was mediated by this signaling pathway. To test this, we first examined the effect of DHA on Akt expression. Treatment of GBM cells with DHA led to a marked decrease in $\mathrm{p}-\mathrm{Akt}^{\mathrm{Ser} 473}$ levels and a gradual decrease in $\mathrm{p}$-mTOR levels.
We also measured AMPK levels, and observed a significant increase in p-AMPK levels after DHA treatment (Fig. 4). These results indicate that DHA suppresses the mTOR pathway in GBM cells through inhibition of Akt and activation of AMPK.

$\omega 3$-PUFAs suppress tumor growth in vivo. To evaluate the relevance of these findings in vivo, we employed fat- 1 tg mice 

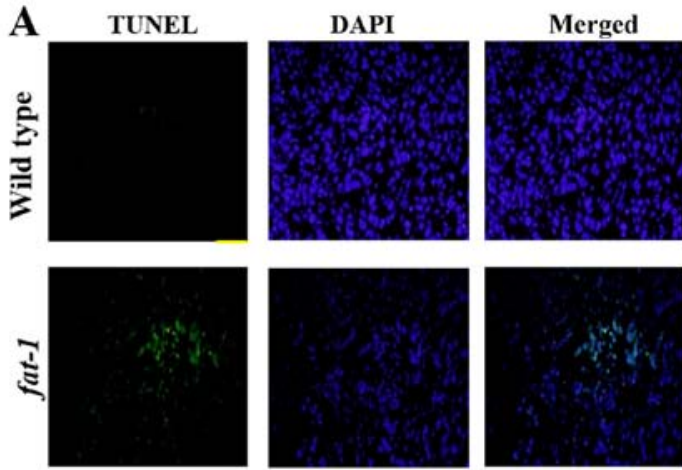

B
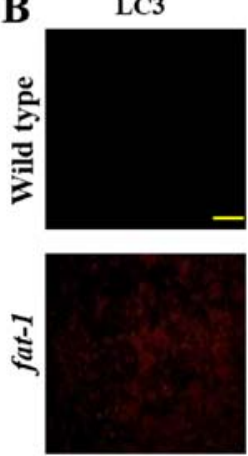

DAPI
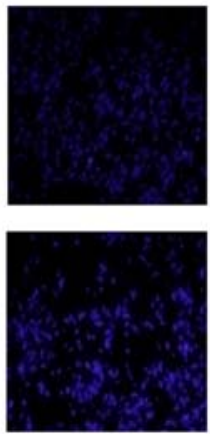

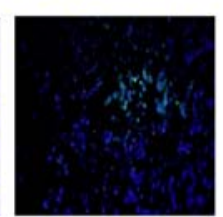

Merged
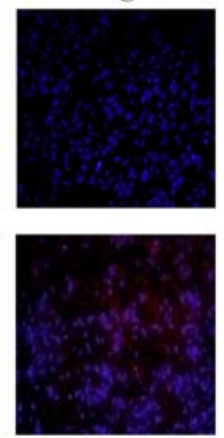
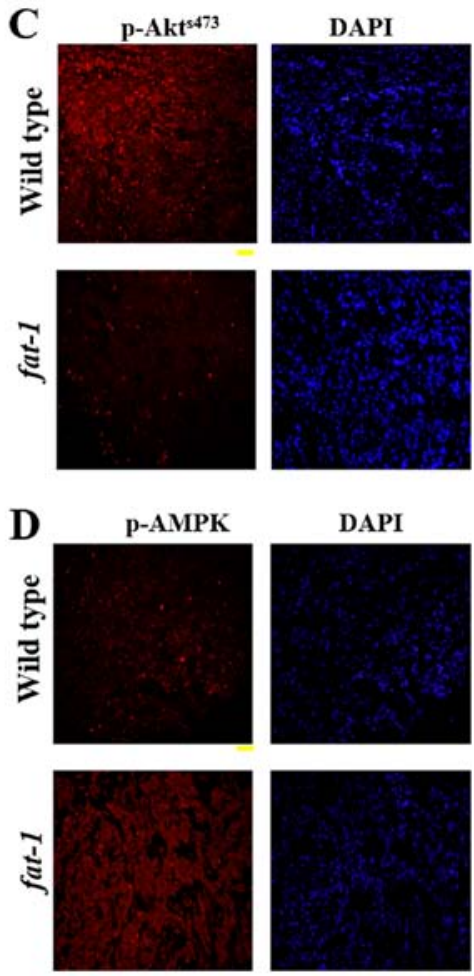

DAPI
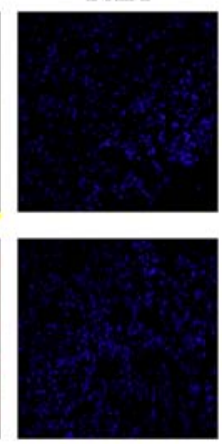
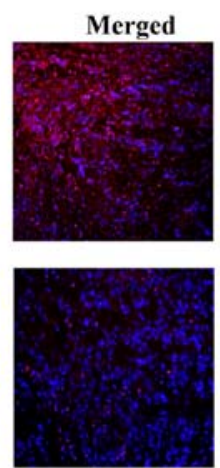

Merged
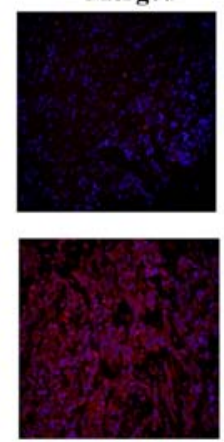

Figure 6. Immunohistochemistry of xenograpft tumor tissues. Representative fluorescence images showing TUNEL assay (A), LC3-II (B), phospho-Akt (Ser473) (C), and phospho-AMPK (D) results, respectively. Fluorescently stained tissues were observed under a fluorescence microscope using DP Controller software (Olympus) for image acquisition.

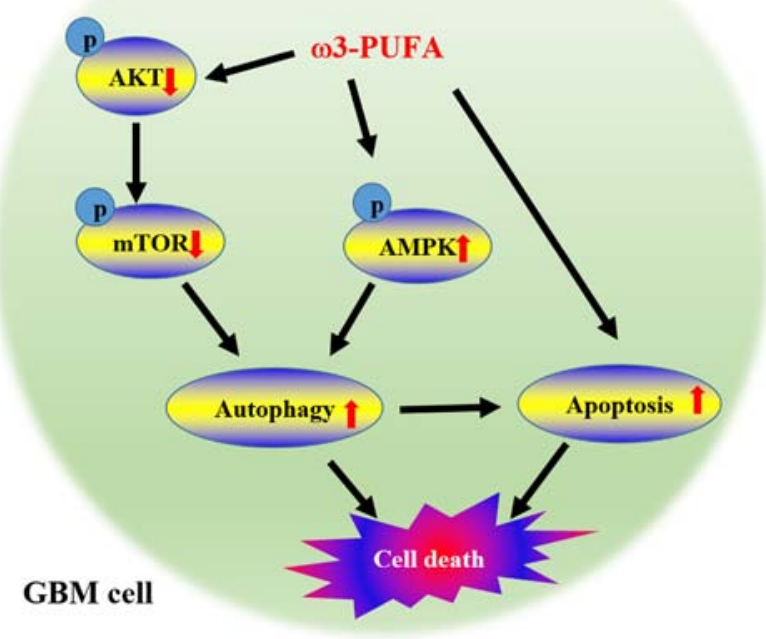

Figure 7. Proposed model of DHA-induced apoptosis and autophagy in GBM cells. DHA-induced autophagy and apoptosis in GBM cells are triggered by inhibition of mTOR activation through Akt inhibition and AMPK activation. GBM, glioblastoma; $\omega 3$-PUFA, omega-3-polyunsaturated fatty acid.

as a model of elevated $\omega 3$-PUFA concentration in tissue. fat- 1 tg mice carry a $\omega 3$-desaturase gene from Caenorhabditis elegans that converts $\omega 6$-PUFAs to $\omega 3$-PUFAs, resulting in a significant increase in the concentration of $\omega 3$-PUFA and a decrease in the concentration of $\omega 6$-PUFAs throughout the body (18). A mouse model of GBM was created using the mouse glioma cell line GL261, which was chosen because this cell line is derived from a GBM from C57BL/6 mice and forms tumors in these mice.

Before implantation of GL261 cells into mice, the effect of DHA on GL261 cells was confirmed in vitro. Consistent with the results obtained in human GBM cells, DHA significantly decreased the viability of GL261 cells (Fig. 5A).

GL261 cells were implanted into fat- 1 tg mice and control mice (C57/BL6 genetic background), and the tumorigenicity of the inoculated cells in these mice was examined. As shown in Fig. 5B, there was a marked difference in tumor volume between fat- $1 \mathrm{tg}(\mathrm{n}=5)$ and control mice $(\mathrm{n}=5)$. After 35 days, the average tumor volume was $75 \%$ lower in the fat-1 tg mice than the tumor volume noted in the control mice (Fig. 5C). To determine whether apoptosis and autophagy were also related to the inhibition of tumor growth observed in vivo, we performed TUNEL assays and measured LC3 levels in the tumor tissues. Both the proportion of TUNEL-positive cells and the level of LC3B were higher in the tumor tissue derived from fat- $1 \mathrm{tg}$ mice than in tumor tissue derived from wild-type mice (Fig. 6A and $\mathrm{B})$, indicating that both apoptosis and autophagy are involved in the reduction of tumor growth in fat- $1 \mathrm{tg}$ mice. Subsequently, to determine whether Akt and AMPK were also related to the increase in apoptosis and autophagy in these mice, the levels of Akt and AMPK in tumor tissue were analyzed by immunohistochemistry. As shown in Fig. 6C and D, the level of p-Akt ${ }^{\mathrm{Ser} 473}$ was reduced and the level of p-AMPK was elevated in tumor tissue derived from fat- 1 tg mice, compared with tumor tissue derived from the control mice. These data demonstrate that $\omega 3$-PUFAs induce both apoptosis and autophagy in vivo by regulating AMPK and Akt/mTOR signaling (Fig. 7). 


\section{Discussion}

Compelling evidence from many studies has demonstrated that DHA induces apoptosis and protects against a variety of cancers, including lung cancer, via multiple targets $(8,13)$. Although the anticancer mechanisms of $\omega 3$-PUFAs in several cancers have been reported, the relevant mechanisms are still unclear in the case of brain cancer. In the present study, we used in vitro experiments and in vivo mouse models to evaluate the anticancer activity of $\omega 3$-PUFAs against GBM. DHA treatment induced dose- and time-dependent growth inhibition in three human GBM cell lines. Of these three cell lines, D54MG cells were selected for further experiments as the viability of D54MG cells was reduced by only $\sim 20 \%$ after DHA treatment. The observations that DHA induced an increase in the concentration of cleaved PARP in D54MG cells, with a concomitant increase in the sub-G1 cell population, as demonstrated by cell cycle analysis using flow cytometry, confirmed that DHA induced apoptotic cell death in D54MG cells (Figs. 1 and 2). Additionally, using the TUNEL assay, a common method for detecting DNA fragmentation resulting from apoptotic signaling cascades, the proportion of TUNELpositive D54MG cells was shown to increase after DHA treatment (Fig. 2B). Gobeil et al (19) reported the possible implication of lysosomal enzymes in the necrotic cleavage of PARP-1. They observed two major PARP-1 fragments (a C-terminal $55 \mathrm{kDa}$ active and a $\mathrm{N}$-terminal $62 \mathrm{kDa}$ inactive fragment) after necrotic inducers like $\mathrm{H}_{2} \mathrm{O}_{2}$ in Jurkat T cells. In our data, only an 89-kDa fragment was observed after DHA treatment (Fig. 2A) in the GBM cells. These results strongly indicate that DHA induced apoptotic cell death, not necrotic cell death in the GBM cells.

Autophagy and apoptosis have been shown to be interconnected by crosstalk between several molecular nodes. The functional relationship between apoptosis and autophagy is complex; in some circumstances, autophagy constitutes a stress adaptation to avoid cell death, whereas in other circumstances, it constitutes an alternative cell death pathway. Both autophagy and apoptosis can be triggered by a common upstream signal (20). Jing et al (13) reported that autophagy and apoptosis occur simultaneously and act cooperatively to induce cell death after DHA treatment. To determine whether autophagy is also induced by DHA in GBM cells, we first examined the levels of LC3-II, an autophagy marker. DHA increased the expression levels of LC3-II in three GBM cell lines (Fig. 3A). These results were confirmed conclusively in D54MG cells transfected with GFP-LC3, which showed a higher number of GFP-LC3 puncta after exposure to DHA (Fig. 3B). Moreover, autophagic flux assays in cells pretreated with chloroquine, a lysosomal inhibitor, confirmed that DHA markedly increased LC3-II expression (Fig. 3C). These results clearly demonstrated that DHA induces GBM cell death by both apoptosis and autophagy.

The Akt/mTOR pathway is one of the most frequently dysregulated pathways in several types of cancers. Several selective inhibitors of Akt and mTOR have been investigated, both in preclinical tumor models and in various clinical trials; however, these inhibitors have been found not to improve survival significantly because of feedback activation of the Akt/mTOR pathway (21). Several other studies have shown that
DHA inhibits the Akt/mTOR pathway in various cancer cells, including prostate cancer cells $(14,22)$. We therefore assessed whether inhibition of $\mathrm{Akt} / \mathrm{mTOR}$ signaling is also related to apoptotic and autophagic cell death in GBM by testing the effect of DHA exposure on Akt and mTOR levels in three GBM cell lines. DHA treatment caused a dose-dependent decrease in $\mathrm{p}-\mathrm{Akt}^{\mathrm{Ser} 473}$ and $\mathrm{p}$-mTOR levels, suggesting that DHA-induced autophagy is related to the inhibition of mTOR through downregulation of p-Akt.

mTOR receives signals from nutrients, growth factors, and many cellular kinases, including AMPK. Phosphorylation of AMPK activates downstream signaling that triggers mTOR inhibition and autophagy $(23,24)$. Jing et al (13) reported that DHA inhibits mTOR through AMPK activation. Our data confirmed that DHA increases the level of p-AMPK and that DHA-induced autophagy is related to inhibition of mTOR through activation of AMPK.

To support these in vitro data, we performed in vivo experiments using fat-1 tg mice, implanting GL261 mouse glioma cells into fat-1 tg mice and wild-type mice. fat-1 tg mice ubiquitously express a $C$. elegans $\omega 3$-desaturase, leading to a significant increase in the $\omega 3$-PUFA/ $\omega 6$-PUFA ratio across all organs and tissues (18). This mouse model was selected as the endogenous source of $\omega 3$-PUFAs provides a consistent $\omega 3$-PUFA concentration and eliminates potential dietary variations associated with long-term feeding of $\omega 3$-PUFAs. A significant reduction in GL261 primary tumor growth was observed in the fat- 1 tg mice (Fig. 5B), and tumor tissue derived from fat 1 tg mice exhibited an increase in the proportion of TUNEL-positive cells (Fig. 6A) and LC3-II levels (Fig. 6B) compared with tumor tissue derived from wild-type mice. These findings provide important in vivo evidence for growth retardation of GL261 cells by $\omega 3$-PUFAs via induction of apoptosis and autophagy. Moreover, we confirmed that p-Akt levels were reduced (Fig. 6C) and p-AMPK levels were elevated (Fig. 6D) in tumor tissue from fat-1 tg mice, compared with tumor tissue from wild-type mice. Thus, the present study clearly demonstrates that $\omega 3$-PUFAs, including DHA, induce GBM cell death through repression of mTOR via Akt inhibition and AMPK activation (Fig. 7). In summary, this study provides important information on the mechanism underlying the antitumorigenic activity of $\omega 3$-PUFAs and encouraging preclinical evidence for a possible strategy for the chemoprevention and treatment of GBM. Therefore, it is possible that $\omega 3$-PUFAs, being non-toxic, may represent safe chemopreventive and therapeutic agents for GBM patients.

\section{Acknowledgements}

This study was supported by the National Research Foundation of Korea (NRF) grant funded by the Korea government (MSIP) (NRF-2015R1D1A1A01056887) and research fund of Chungnam National University (2014). We thank Dr Jing X. Kang at the Harvard Medical School for providing the Fat-1 transgenic mice.

\section{References}

1. Siegel RL, Miller KD and Jemal A: Cancer Statistics, 2017. CA Cancer J Clin 67: 7-30, 2017.

2. Holland EC: Glioblastoma multiforme: The terminator. Proc Natl Acad Sci USA 97: 6242-6244, 2000. 
3. Preusser M, de Ribaupierre S, Wöhrer A, Erridge SC, Hegi M, Weller M and Stupp R: Current concepts and management of glioblastoma. Ann Neurol 70: 9-21, 2011.

4. Simopoulos AP: Human requirement for $\mathrm{N}-3$ polyunsaturated fatty acids. Poult Sci 79: 961-970, 2000.

5. Simopoulos AP: Omega-3 fatty acids in inflammation and autoimmune diseases. J Am Coll Nutr 21: 495-505, 2002.

6. Simopoulos AP: The importance of the ratio of omega-6/ omega- 3 essential fatty acids. Biomed Pharmacother 56 365-379, 2002.

7. Song KS, Jing K, Kim JS, Yun EJ, Shin S, Seo KS, Park JH, Heo JY, Kang JX, Suh KS, et al: Omega-3-polyunsaturated fatty acids suppress pancreatic cancer cell growth in vitro and in vivo via downregulation of Wnt/Beta-catenin signaling. Pancreatology 11: 574-584, 2011.

8. Lim K, Han C, Dai Y, Shen M and Wu T: Omega-3 polyunsaturated fatty acids inhibit hepatocellular carcinoma cell growth through blocking beta-catenin and cyclooxygenase-2. Mol Cancer Ther 8: 3046-3055, 2009.

9. Lim K, Han C, Xu L, Isse K, Demetris AJ and $\mathrm{Wu} T$ : Cyclooxygenase-2-derived prostaglandin E2 activates betacatenin in human cholangiocarcinoma cells: Evidence for inhibition of these signaling pathways by omega 3 polyunsaturated fatty acids. Cancer Res 68: 553-560, 2008.

10. Jeong S, Jing K, Kim N, Shin S, Kim S, Song KS, Heo JY, Park JH, Seo KS, Han J, et al: Docosahexaenoic acid-induced apoptosis is mediated by activation of mitogen-activated protein kinases in human cancer cells. BMC Cancer 14: 481, 2014.

11. Yun EJ, Song KS, Shin S, Kim S, Heo JY, Kweon GR, Wu T, Park JI and Lim K: Docosahexaenoic acid suppresses breast cancer cell metastasis by targeting matrix-metalloproteinases. Oncotarget 7: 49961-49971, 2016.

12. Kim N, Jeong S, Jing K, Shin S, Kim S, Heo JY, Kweon GR, Park SK, Wu T, Park JI, et al: Docosahexaenoic acid induces Cell death in human non-small cell lung cancer cells by repressing mTOR via AMPK activation and PI3K/Akt inhibition. Biomed Res Int 2015: 239764, 2015.

13. Jing K, Song KS, Shin S, Kim N, Jeong S, Oh HR, Park JH, Seo KS, Heo JY, Han J, et al: Docosahexaenoic acid induces autophagy through $\mathrm{p} 53 / \mathrm{AMPK} / \mathrm{mTOR}$ signaling and promotes apoptosis in human cancer cells harboring wild-type p53. Autophagy 7: 1348-1358, 2011.
14. Shin S, Jing K, Jeong S, Kim N, Song KS, Heo JY, Park JH, Seo KS, Han J, Park JI, et al: The omega-3 polyunsaturated fatty acid DHA induces simultaneous apoptosis and autophagy via mitochondrial ROS-mediated Akt-mTOR signaling in prostate cancer cells expressing mutant p53. BioMed Res Int 2013: $568671,2013$.

15. Jing K, Shin S, Jeong S, Kim S, Song KS, Park JH, Heo JY, Seo KS, Park SK, Kweon GR, et al: Docosahexaenoic acid induces the degradation of HPV E6/E7 oncoproteins by activating the ubiquitin-proteasome system. Cell Death Dis 5: e1524, 2014.

16. Zajdel A, Wilczok A, Latocha M, Tarkowski M, Kokocińska M and Dzierzewicz Z: Polyunsaturated fatty acids potentiate cytotoxicity of cisplatin in A549 cells. Acta Pol Pharm 71: 1060-1065, 2014.

17. Gleissman H, Johnsen JI and Kogner P: Omega-3 fatty acids in cancer, the protectors of good and the killers of evil? Exp Cell Res 316: 1365-1373, 2010.

18. Kang JX, Wang J, Wu L and Kang ZB: Transgenic mice: Fat-1 mice convert n-6 to n-3 fatty acids. Nature 427: 504, 2004.

19. Gobeil S, Boucher CC, Nadeau D and Poirier GG: Characterization of the necrotic cleavage of poly(ADP-ribose) polymerase (PARP-1): Implication of lysosomal proteases. Cell Death Differ 8: 588-594, 2001

20. Maiuri MC, Zalckvar E, Kimchi A and Kroemer G: Self-eating and self-killing: Crosstalk between autophagy and apoptosis. Nat Rev Mol Cell Biol 8: 741-752, 2007.

21. Jhanwar-Uniyal M, Albert L, McKenna E, Karsy M, Rajdev P, Braun A and Murali R: Deciphering the signaling pathways of cancer stem cells of glioblastoma multiforme: Role of Akt/ mTOR and MAPK pathways. Adv Enzyme Regul 51: 164-170, 2011.

22. Schley PD, Jijon HB, Robinson LE and Field CJ: Mechanisms of omega-3 fatty acid-induced growth inhibition in MDA-MB-231 human breast cancer cells. Breast Cancer Res Treat 92: 187-195, 2005.

23. Faivre S, Kroemer G and Raymond E: Current development of mTOR inhibitors as anticancer agents. Nat Rev Drug Discov 5: 671-688, 2006.

24. Shaw RJ: Glucose metabolism and cancer. Curr Opin Cell Biol 18: 598-608, 2006. 